\title{
Free vibration of rectangular nanoplate strips
}

\author{
Jaan Lellep ${ }^{1}$, Mainul Hossain ${ }^{2}$ \\ Institute of Mathematics and Statistic, University of Tartu, Tartu, Estonia \\ E-mail: 1jaan.lellep@ut.ee (corresponding author)
}

\begin{abstract}
Natural vibrations of nanobeams and nanosheets are investigated with the help of nonlocal theories of elasticity. The vibration analysis is based on the size-dependent non-local theory of elasticity developed by A. C. Eringen. It is assumed that the nano-structures under consideration have rectangular cross sections with piece wise constant dimensions and that the nanoplates are weakened with defects. The influence of the crack on the vibration of the nanoplate is assessed with the aid of additional local compliance developed in previous papers. Numerical results are presented for one- and two-stepped nanoplates.
\end{abstract}

Keywords: crack, nanoplate, vibration, eigenfrequency, nonlocal elasticity.

\section{Introduction}

It is well known that the classical continuum mechanics which resorts to the Hooke's law can not predict the size effect necessary for adequate modelling of the material behavior in the nanomechanics. Among the size-dependent theories the concept of the nonlocal elasticity developed by Eringen (2002) has an outstanding place. This theory is employed in the present paper. The nonlocal elasticity has been used to study the lattice dispersion of elastic waves, wave propagation in composites, also the dislocation mechanics and fracture mechanics (Reddy, 2007; Wang \& Liew, 2007). The buckling and vibration of carbon nanotubes based on nonlocal theories was studied by Wang and Varadan (2006), Zhang, Liu, and Xie (2005).

In the present paper the natural vibrations of nanobeams and nanosheets will be studied in the frame work of the plate theory accounting for the inertia of rotation. The plate strips under consideration have piece wise constant thickness and are weakened with stable cracks.

\section{Problem formulation}

Natural vibrations of a stepped nanoplate strip stretched with the axial force $N$ are studied. The nanoplate strip has rectangular cross section with constant width $b$ and height

$$
h=\left\{\begin{array}{lc}
h_{0} & x \in\left(0, a_{1}\right) \\
h_{1} & x \in\left(a_{1}, a_{2}\right), \\
h_{2} & x \in\left(a_{2}, l\right)
\end{array}\right.
$$

where $a_{1}, a_{2}, h_{0}, h_{1}, h_{2}$ are given constants and $a_{1}<a_{2}$.

The system of coordinates is introduced so that the coordinate axis $0 x$ coincides with the axis of the straight nanoplate strip and its origin is located at the center of the left-hand edge of the nanoplate strip. The stepped nanoplate of piece wise constant height has defects at the cross sections $x=a_{1}$ and $x=a_{2}$. The defects are treated herein as stable cracks of length $c_{1}$ and $c_{2}$ respectively. In the solid mechanics it is accepted that the presence of the crack causes the change of the internal (potential) energy of the nanoelements. This change is interpreted as the occurring of the additional flexibility caused by the crack. In the present paper we shall determine the frequencies of natural vibrations of nanoplate strips of piece wise constant thickness. The nanoplate strips are weakened by cracks which are located at the corners of steps. The aim of the study is to elucidate the dependence of eigenfrequencies on the parameters $a_{1}, a_{2}, h_{0}, h_{1}, h_{2}$ also on the physical parameters.

(C) 2019 Authors. Published by VGTU Press. This is an open-access article distributed under the terms of the Creative Commons Attribution (http://creativecommons.org/licenses/by/4.0/) License, which permits unrestricted use, distribution, and reproduction in any medium, provided the original author and source are credited. 


\section{Equation of motion}

The main equations of a beam or plate problem consist of geometrical relations, equilibrium equations and constitutive equations with boundary conditions. A geometrically non-linear beam model accounting for the effect of the inertia of rotation will be used in the present study. It is reasonable to assume that the axial force $N$ is constant throughout the nanoplate strip. Following the sources (Reddy, 2007; Soedel, 2004; Lellep \& Kägo, 2011) we shall use the equilibrium equation in the form

$$
\frac{\partial^{2} M}{\partial x^{2}}+N \frac{\partial^{2} W}{\partial x^{2}}=\mu \frac{\partial^{2} W}{\partial t^{2}}-I_{r} \frac{\partial^{4} W}{\partial x^{2} \partial t^{2}} .
$$

In (2) and henceforth $M(x, t)$ denotes the bending moment, $W(x, t)$ the transverse displacement, $t$ being current time instant. The parameter $\mu$ in (2) stands for the mass per unit length of the nanoplate and $I_{r}$ is the rotarory inertia of the current cross-section. Since the height of the nanoplate strip is according to (1) piece wise constant the mass per unit length of the nanobeam can be presented as

$$
\mu= \begin{cases}\mu_{0} & x \in\left(0, a_{1}\right), \\ \mu_{1} & x \in\left(a_{1}, a_{2}\right), \\ \mu_{2} & x \in\left(a_{2}, l\right) .\end{cases}
$$

Similarly, the moment of inertia is presented as

$$
I=\left\{\begin{array}{cc}
I_{0} & x \in\left(0, a_{1}\right), \\
I_{1} & x \in\left(a_{1}, a_{2}\right), \\
I_{2} & x \in\left(a_{2}, l\right) .
\end{array}\right.
$$

In (3) $\mu_{0}=\rho h_{0}, \mu_{1}=\rho h_{1}, \mu_{2}=\rho h_{2}$ where $\rho$ is the density of the material. The intertial moments $I_{0}, I_{1}, I_{2}$ can be calculated as

$$
I_{0}=\frac{b h_{0}^{3}}{12}, I_{1}=\frac{b h_{1}^{3}}{12}, I_{2}=\frac{b h_{2}^{3}}{12} .
$$

It can be observed from (2) that in the case $I_{r}=0$ the equilibrium Eq. (2) coincides with the classical non-linear equation of beam and plate dynamics (Lellep \& Kägo, 2011; Lellep \& Lenbaum, 2018). It is well known that the vibration of nanobeams can be prescribed with the nonlocal theory of elasticity. Probably the simpliest case of the constitutive equation can be presented as

$$
M-\eta \frac{\partial^{2} M}{\partial x^{2}}-M_{E}=0,
$$

where $M_{E}$ is the bending moment in the classical theory of elasticity (Roostai \& Haghpanahi, 2016; Lellep \& Lenbaum, 2018; Wang \& Liew, 2007). It is well known that in the Euler-Bernoulli theory the bending moment is defined as

$$
M_{E}=-E I \frac{\partial^{2} W}{\partial x^{2}},
$$

where $E$ stands for the Young modulus. The parameter $\eta$ in (6) is a material constant calculated as $\eta=\left(e_{0} a\right)^{2}, e_{0}$ and $a$ being the lattice parameters of the material, as shown in (Roostai \& Haghpanahi, 2016). Thus, (6) and (7) yield

$$
M-\eta \frac{\partial^{2} M}{\partial x^{2}}+E I \frac{\partial^{2} W}{\partial x^{2}}=0 .
$$

Combining the Eqs (2), (6) and (8) one obtains

$$
M=-E I W^{\prime \prime}+\eta\left(-N W^{\prime \prime}+\mu \ddot{W}+I_{r} \ddot{W}^{\prime \prime}\right),
$$

where dots denote the differentiation with respect to time and prims with respect to the variable $x$. 
Differentiating (9) twice with respect to $x$ gives

$$
M^{\prime \prime}=-(E I+\eta N) W^{I V}+\eta\left(\mu \ddot{W}^{\prime \prime}-I_{r} \ddot{W}^{I V}\right),
$$

where $\mu, \eta, E$ are constants.

\section{Solution of the equation of motion}

The equation of motion takes the form

$$
(E I+\eta N) W^{I V}+N W^{\prime \prime}+\eta\left(-\mu \ddot{W}^{\prime \prime}+I_{r} \ddot{W}^{I V}\right)+\mu \ddot{W}+I_{r} \ddot{W}^{\prime \prime}=0,
$$

where $\mu$ and $I$ are defined by (3) and (4).

The solution of (11) can be obtained assuming that

$$
W=w(x) e^{i \omega_{c} t}
$$

where $w(x)$ depends on $x$ only and $e^{i \omega_{c} t}$ is a function of time $t, \omega_{c}$ is the dimensional frequency.

The dimensionless variables can be defined as follows

$$
\begin{gathered}
x=\frac{X}{l}, w=\frac{W}{l}, \varepsilon=\frac{e_{0} a}{l}, n=\frac{N l^{2}}{E I}, \omega=\omega_{c} l^{2} \sqrt{\frac{\rho h_{0}}{E I}}, \mu=\rho h_{0}, r_{0}=\frac{1}{12} \frac{h_{0}^{2}}{l^{2}} ; \\
\gamma_{1}=\frac{h_{1}}{h_{0}}, \omega_{1}=\gamma_{1} \omega, \gamma_{2}=\frac{h_{2}}{h_{0}}, \omega_{2}=\gamma_{2} \omega,
\end{gathered}
$$

where $\omega$ is the nondimensional frequency.

For $x \in\left(0, a_{1}\right)$ the equation of motion is simplified in the form

$$
\left(1+\varepsilon^{2} n-r_{0} \omega^{2}\right) w^{I V}+\left(\varepsilon^{2} \omega^{2}-n+r_{0} \omega^{2}\right) w^{\prime \prime}-\omega^{2} w=0
$$

Similarly one has

$$
\left(1+\varepsilon^{2} n-r_{1} \omega_{1}^{2}\right) w^{I V}+\left(\varepsilon^{2} \omega_{1}^{2}-n+r_{1} \omega_{1}^{2}\right) w^{\prime \prime}-\omega_{1}^{2} w=0
$$

for $x \in\left(a_{1}, a_{2}\right)$ and

$$
\left(1+\varepsilon^{2} n-r_{2} \omega_{2}^{2}\right) w^{I V}+\left(\varepsilon^{2} \omega_{2}^{2}-n+r_{2} \omega_{2}^{2}\right) w^{\prime \prime}-\omega_{2}{ }^{2} w=0
$$

for $x \in\left(a_{2}, l\right)$.

For solution of the Eq. (14) one has to solve the characteristic equation

$$
\left(1+\varepsilon^{2} n-r_{0} \omega^{2}\right) \lambda_{0}^{4}+\left(\varepsilon^{2} \omega^{2}-n+r_{0} \omega^{2}\right) \lambda_{0}^{2}-\omega^{2}=0 .
$$

The characteristic equations corresponding to (15) and (16) are

$$
\left(1+\varepsilon^{2} n-r_{1} \omega_{1}^{2}\right) \lambda_{1}^{4}+\left(\varepsilon^{2} \omega_{1}^{2}-n+r_{1} \omega_{1}^{2}\right) \lambda_{1}^{2}-\omega_{1}^{2}=0
$$

and

$$
\left(1+\varepsilon^{2} n-r_{2} \omega_{2}^{2}\right) \lambda_{2}^{4}+\left(\varepsilon^{2} \omega_{2}^{2}-n+r_{2} \omega_{2}^{2}\right) \lambda_{2}^{2}-\omega_{2}^{2}=0
$$

Evidently, the roots of the characteristic equation for (17) in the seqment $\left(0, a_{1}\right)$ are

$$
\left(\lambda_{0}\right)_{1,2}= \pm i \theta_{0}
$$

and 


$$
\left(\lambda_{0}\right)_{3,4}= \pm \beta_{0}
$$

where $\theta_{0}$ and $\beta_{0}$ are real numbers. In the similar way one can solve the rest equations in the system (18), (19).

It can be rechecked that the general solutions of the Eqs (17)-(19) can be presented for $x \in\left(0, a_{1}\right)$ as

$$
w=A_{1} \cosh \left(\theta_{0} x\right)+A_{2} \sinh \left(\theta_{0} x\right)+A_{3} \cos \left(\beta_{0} x\right)+A_{4} \sin \left(\beta_{0} x\right)
$$

for $x \in\left(a_{1}, a_{2}\right)$ as

$$
w=B_{1} \cosh \left(\theta_{1} x\right)+B_{2} \sinh \left(\theta_{1} x\right)+B_{3} \cos \left(\beta_{1} x\right)+B_{4} \sin \left(\beta_{1} x\right)
$$

for $x \in\left(a_{2}, l\right)$ as

$$
w=D_{1} \cosh \left(\theta_{2} x\right)+D_{2} \sinh \left(\theta_{2} x\right)+D_{3} \cos \left(\beta_{2} x\right)+D_{4} \sin \left(\beta_{2} x\right),
$$

where $A_{1}-A_{4}, B_{1}-B_{4}, D_{1}-D_{4}$ stand for arbitrary constants.

Those constants will be defined with the help of corresponding boundary and intermediate requirements. For instance, in the case of a simply supported edge the transverse displacement $w$ and the bending moment $M$ must vanish. Thus, in the case of a simply supported edge at $x=0$

$$
w(0)=0, M(0)=0 .
$$

Note that similar requirement will be obtained for the right hand end of the beam or strip also. At $x=l$ one has

$$
w(l)=0, M(l)=0 .
$$

\section{Additional compliance due to the crack}

It seems that the defects occurring in manufacturing and operation of structures are practically unavoidable sources of additional compliance. Let us denote the compliance matrix by $C$ and its elements by $C_{i j}$. Evidently (Anderson, 2017; Lellep \& Lenbaum, 2018; Lellep \& Liyvapuu, 2016)

$$
C_{i j}=\frac{\partial u_{i}}{\partial P_{j}},
$$

where $u_{i}$ is a displacement and $P_{j}$ a generalized external force.

In the present case the bending moment $M$ serves as the generalized force. Thus instead of the matrix we have a single function of compliance. Denote the change of the slope as

$$
\theta=w^{\prime}(a+0, t)-w^{\prime}(a-0, t) \text {. }
$$

Thus, one can expect that

$$
\theta=C M(a, t) .
$$

Dimarogonas (1996) and other authors have developed the methods for determination of the additional compliance. This enables to determine the frequencies of natural vibrations of nanobeams and nanostrips. This concept was extended to curved arches by Lellep and Liyvapuu (2016) and to stepped plate strips by Lellep and Kägo (2011).

\section{Numerical results}

The results are presented for simply supported one- and two-stepped plate strips with cracks in Figures 2-7. In the case of two-stepped plate strips, steps are placed in equal distance from the center of the strip.
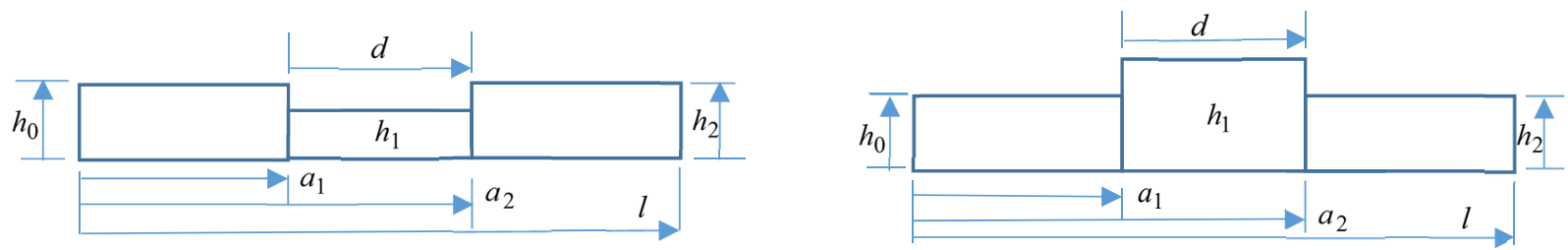

Figure 1. Geometry of stepped nanoplate strips 
The natural frequencies of nanoplate strips with a unit step are depicted in Figures $2-7$, where $\varepsilon=0.5$. In Figure 1 the geometry of nanoplate strip is illustrated in the case where strip is divided into three spans with steps and cracks. In Figure 2 the relationship between the natural frequency and the heights ratio of the nanoplate strip is shown for uniform nanoplates without any defects. The height of plate is piece wise constant. In Figure 3 the natural frequency is presented for different crack depth and step length.

In Figures 4-6 the dependence of the natural frequency on the step location and the rotatory inertia is shown. Here rotatory inertia depends on the height of the plate strip. The Figures 4-6 reveal the matter that rotatory inertia diminish the natural frequency and it is very sensitive at the higher mode of frequency.

In Figure 7 demonstrates the natural frequency versus axial load for different stepped span length. It is understandable from the figure that compressive axial load increases frequency. On the other hand, the tensile axial load decreases frequency.

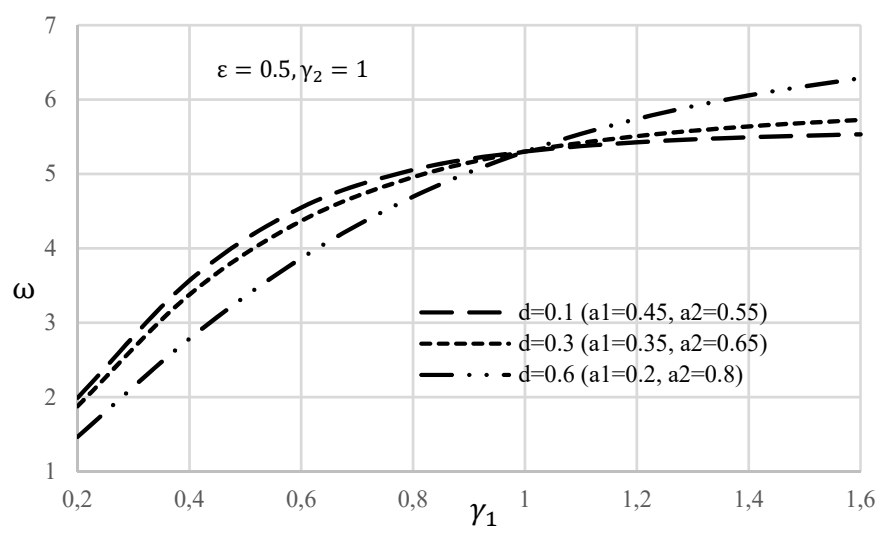

Figure 2. Natural frequency versus heights ratio

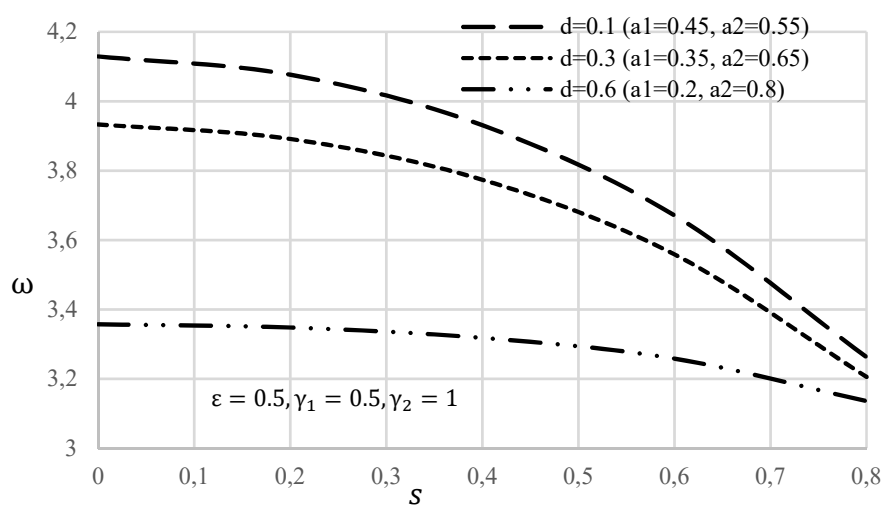

Figure 3. Natural frequency versus crack length

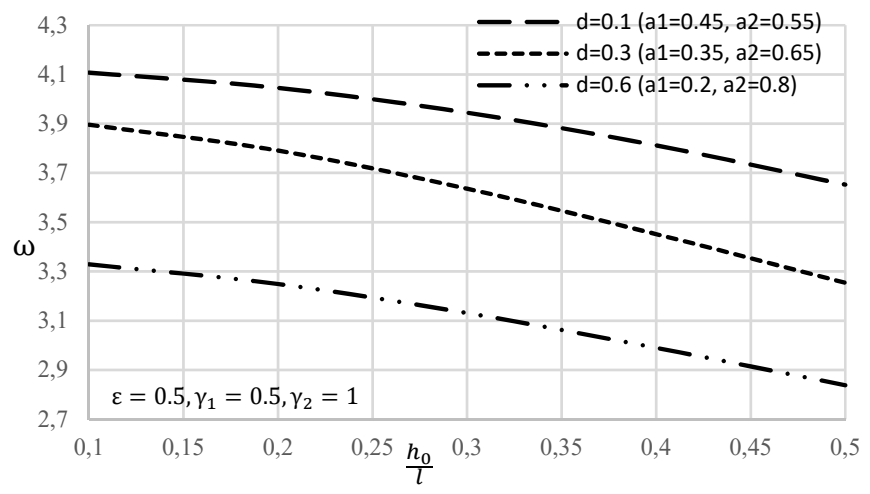

Figure 4. First natural frequency versus height length ratio 


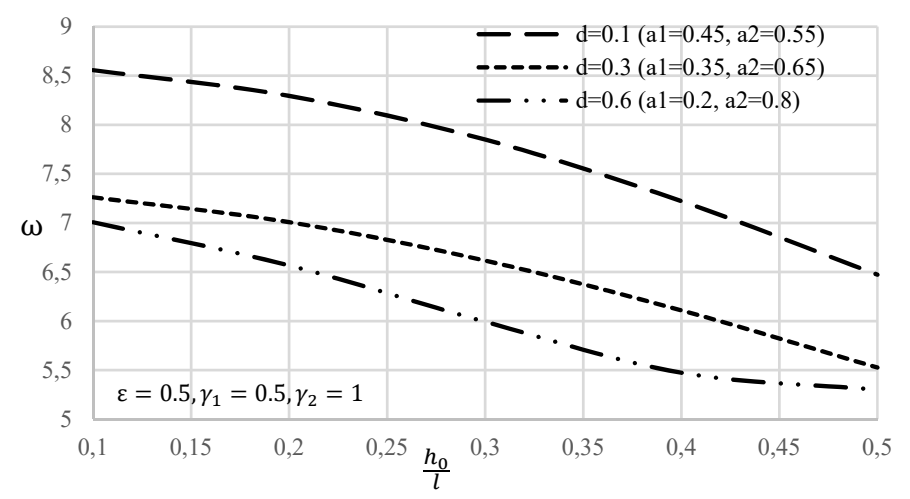

Figure 5. Second natural frequency versus height length ratio

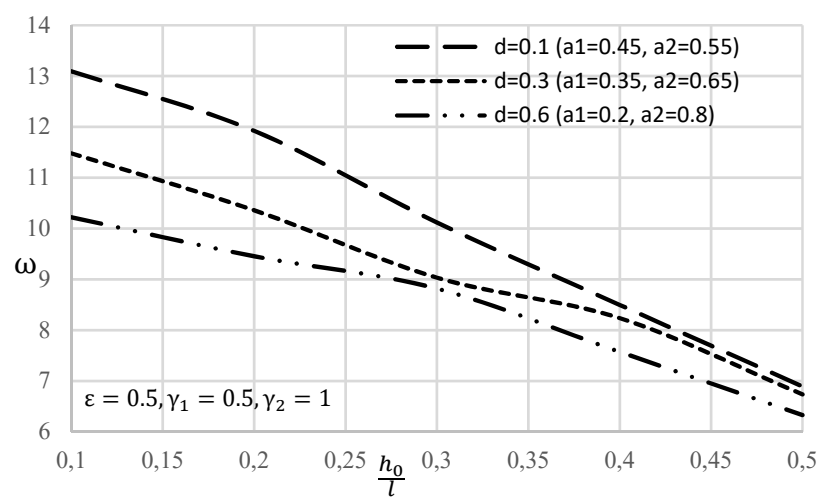

Figure 6. Third natural frequency versus height length ratio

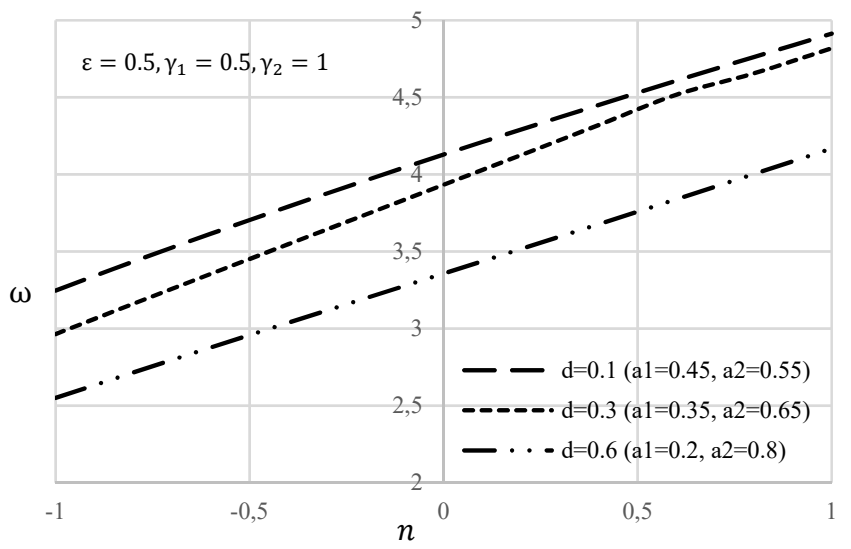

Figure 7. Natural frequency versus axial load

\section{Concluding remarks}

A method for determination of natural frequencies of stepped nanobeams and nanosheets has been developed in the present paper. The current approach is based on the nonlocal theory of elasticity. The equibrium equations account for the influence of the rotational inertia. The calculations are carried out for one-stepped, also two-stepped nanoplate strips. The results of calculations are in good correlation with similar results of other researchers in the case of nanobeams with constant dimensions.

\section{Acknowledgements}

The partial support from the Institutional Research Funding IUT2057 of Estonian Ministry of Education and Research is gratefully acknowledged. Also, the support from Estonian Doctoral School in Mathematics is acknowledged. 


\section{References}

Anderson, T. L. (2017). Fracture mechanics. Boca Raton: CRC Press. https://doi.org/10.1201/9781315370293

Dimarogonas, A. D. (1996). Vibration of cracked structures: A state of the art review. Engineering Fracture Mechanics, 55, 831857. https://doi.org/10.1016/0013-7944(94)00175-8

Eringen, A. C. (2002). Nonlocal continuum field theories. New York: Springer.

Lellep, J., \& Kägo, E. (2011). Vibration of elastic stretched strips with cracks. International Journal of Mechanics, 5, 27-34.

Lellep, J., \& Lenbaum, A. (2018). Free vibrations of stepped nanobeams. International Journal of Computational Methods and Experimental Measurements, 6(4), 716-725. https://doi.org/10.2495/CMEM-V6-N4-716-725

Lellep, J., \& Liyvapuu, A. (2016). Natural vibrations of stepped arches with cracks. Agronomy Research, 14, 821-830.

Reddy, J. N. (2007). Nonlocal theories for bending, buckling and vibration of beams. International Journal of Engineering Science, 45(2-8), 288-307. https://doi.org/10.1016/j.ijengsci.2007.04.004

Roostai, H., \& Haghpanahi, M. (2016). Vibration of nanobeams of different boundary conditions with multiple cracks based on nonlocal elasticity theory. Applied Mathematical Modelling, 38(3), 1155-1169. https://doi.org/10.1016/j.apm.2013.08.011

Soedel, W. (2004). Vibration of shells and plates. New York: Marcel Dekker.

Wang, Q., \& Liew, K. M. (2007). Application of nonlocal continuum mechanics to static analysis of micro- and nano-structures. Physics Letters A, 363(3), 236-242. https://doi.org/10.1016/j.physleta.2006.10.093

Wang, Q., \& Varadan, V. K. (2006). Vibration of carbon nanotubes studied using nonlocal continuum mechanics. Smart Materials and Structures, 15, 659-666. https://doi.org/10.1088/0964-1726/15/2/050

Zhang, Y. Q., Liu, G. R., \& Xie, X. Y. (2005). Free transverse vibrations of double-walled carbon nanotubes using a theory of nonlocal elasticity. Physical Review B, 71, 195404. https://doi.org/10.1103/PhysRevB.71.195404 